\title{
A DECOMPOSITION OF INTEGER VECTORS. IV
}

\author{
A. SCHINZEL \\ (Received 10 May 1989) \\ Communicated by J. H. Loxton \\ In memory of Kurt Mahler
}

\begin{abstract}
Given $m$ linearly independent vectors $\mathbf{n}_{1}, \ldots, \mathbf{n}_{m} \in \mathbf{Z}^{k}$ and an integer $l \in[m, k]$ one proves the existence of $l$ linearly independent vectors $\mathbf{p}_{1}, \ldots, \mathbf{p}_{l} \in \mathbf{Z}^{k}$ or $\mathbf{q}_{1}, \ldots, \mathbf{q}_{l} \in \mathbf{Z}^{k}$ of small size (suitably measured) such that the $n_{i}$ 's are linear combinations of $\mathbf{p}_{j}$ 's with rational coefficients or of $\mathbf{q}_{j}$ 's with integer coefficients.
\end{abstract}

1980 Mathematics subject classification (Amer. Math. Soc.) (1985 Revision): 11 H 41.

In order to generalize the results of [10] (Part III of this series) let us introduce the following notation. Given $m$ linearly independent vectors $\mathbf{n}_{1}, \ldots, \mathbf{n}_{m} \in$ $\mathbb{Z}^{k}$ let $H\left(\mathbf{n}_{1}, \mathbf{n}_{2}, \ldots, \mathbf{n}_{m}\right)$ denote the maximum of the absolute values of all minors of order $m$ of the matrix

$$
\left(\begin{array}{c}
\mathbf{n}_{1} \\
\vdots \\
\mathbf{n}_{m}
\end{array}\right)
$$

and $D\left(\mathbf{n}_{1}, \ldots, \mathbf{n}_{m}\right)$ the greatest common divisor of these minors. Furthermore, let

$$
h(\mathbf{n})=H(\mathbf{n}) \quad \text { for } \mathbf{n} \neq \mathbf{0}, \quad h(\mathbf{0})=0 .
$$

(C) 1991 Australian Mathematical Society 0263-6115/91 \$A2.00+0.00 
Definition 1. For $k \geq l \geq m, k>m$, let

$$
\begin{aligned}
& c_{0}(k, l, m)=\operatorname{supinf}\left(\frac{D\left(\mathbf{n}_{1}, \ldots, \mathbf{n}_{m}\right)}{H\left(\mathbf{n}_{1}, \ldots, \mathbf{n}_{m}\right)}\right)^{\frac{k-l}{k-m}} \prod_{i=1}^{l} h\left(\mathbf{p}_{i}\right), \\
& c_{1}(k, l, m)=\operatorname{supinf}\left(\frac{D\left(\mathbf{n}_{1}, \ldots, \mathbf{n}_{m}\right)}{H\left(\mathbf{n}_{1}, \ldots, \mathbf{n}_{m}\right)}\right)^{\frac{k-l}{k-m}} \prod_{i=1}^{l} h\left(\mathbf{q}_{i}\right),
\end{aligned}
$$

where the supremum is taken over all sets of linearly independent vectors $\mathbf{n}_{1}, \ldots, \mathbf{n}_{m} \in \mathbb{Z}^{k}$ and the infimum is taken over all sets of linearly independent vectors $\mathbf{p}_{1}, \ldots, \mathbf{p}_{l} \in \mathbb{Z}^{k}$ or $\mathbf{q}_{1}, \ldots, \mathbf{q}_{l} \in \mathbb{Z}^{k}$ such that for all $i \leq m$,

$$
\mathbf{n}_{i}=\sum_{j=1}^{l} u_{i j} \mathbf{p}_{j}, \quad u_{i j} \in \mathbb{Q}, \quad \mathbf{n}_{i}=\sum_{j=1}^{l} u_{i j} \mathbf{q}_{j}, \quad u_{i j} \in \mathbb{Z} .
$$

The Bombieri-Vaaler refinement [1] of the Siegel lemma easily leads (on the lines of the proof of (8) in [10]) to the conclusion that $c_{0}(k, l, m)$ is finite, first obtained by Yu. Teterin. The aim of this paper is to give bounds for $c_{0}(k, l, m)$ and $c_{1}(k, l, m)$ which are independent of $k$. First however we shall introduce three further series of constants, this time of geometric character.

Definition 2. For a given positive integer $m$, let $\kappa_{m}$ be the volume of the unit ball in $\mathbb{R}^{m}$,

$$
g_{0}(m)=\operatorname{supinf} \frac{\operatorname{vol} \mathbb{P}}{\operatorname{vol} \mathbb{K}}, \quad g_{1}(m)=\operatorname{supinf} \frac{\operatorname{vol} \mathbb{P}}{\operatorname{vol} \mathscr{E}(\mathbb{K})} \cdot \frac{\kappa_{m}}{2^{m}},
$$

where the suprema are taken over all $m$-dimensional convex bodies $\mathbb{K}$ situated in $\mathbb{R}^{m}$, symmetric with respect to the origin, the infima are taken over all parallelopipeds containing $\mathbb{K}$ symmetric with respect to the origin and $\mathscr{E}(\mathbb{K})$ denotes the ellipsoid of the maximum volume contained in $\mathbb{K}$. (It is unique; see [7].) Clearly

$$
\frac{2^{m}}{\kappa_{m}} \leq g_{0}(m) \leq \frac{2^{m}}{\kappa_{m}} g_{1}(m) .
$$

The best published result pertaining to $g_{0}(m), g_{1}(m)$ seems to be the following inequality due to Dvoretzky and Rogers [4, Theorem 5A]:

$$
g_{1}(m) \leq\left(\frac{m^{m}}{m !}\right)^{1 / 2}
$$

Professor A. Pelczyński who indicated to me the paper [4] has improved the above inequality by showing together with S. J. Szarek that (see [9, Proposition 2.1])

$$
g_{1}(m)^{2} \leq\left(\begin{array}{c}
\frac{m(m+1)}{2} \\
m
\end{array}\right)\left(\frac{2}{m+1}\right)^{m}
$$


and, on the other hand, they have proved that (ibid., Section 6)

$$
g_{1}(m)^{2} \geq \frac{2 m}{m+1}
$$

For $m \leq 2$ the two bounds coincide and give

$$
g_{1}(1)=1, g_{1}(2)=\sqrt{\frac{4}{3}}
$$

According to [9, Theorem 5.1], for every $\varepsilon>0$,

$$
\log g_{1}(m)=\frac{m}{2}+o\left(m^{\frac{2}{3}+\varepsilon}\right) .
$$

I am indebted to Professor Pelczyński also for the paradigm (for $l=2$ ) of the proof of Lemma 1 below, which he has since proved in another way (see [9], Corollary 3.1).

We shall prove

THEOREM 1. For all integers $k, l, m$ satisfying $k \geq l \geq m, k>m>0$,

$$
\begin{aligned}
c_{0}(k, l, m) \leq \min \left\{(l-m+1)^{l / 2} g_{1}(m) \gamma_{l}^{l / 2}, \frac{l !}{m !} g_{0}(m),\right. \\
\\
\left.\left(\begin{array}{c}
l \\
m
\end{array}\right)^{l / 2} l^{(l-m) / 2} g_{1}(l) \gamma_{l}^{l / 2}\right\},
\end{aligned}
$$

where $\gamma_{l}$ is the Hermite constant. For $l=m \leq 2$ we have here equality.

THEOREM 2. For all integers $k, k, m$ satisfying $k \geq l \geq m, k>m>0$ we have

$$
\frac{c_{1}(k, l, m)}{c_{0}(k, l, m)} \leq f(l)=\sup _{\mathbf{A}} \inf _{\mathbf{U}}\left(\sum_{j=1}^{l}\left|\delta_{i j}\right|\right),
$$

where $\left[\delta_{i j}\right]=\mathbb{U A} A^{-1}, \mathbf{A}$ and $\mathbb{U}$ run through all lower triangular non-singular integral matrices and all lower triangular unimodular integral matrices of order $l$, respectively. Moreover

$$
f(l) \leq \frac{(l+\lambda+1) !}{4^{l-\lambda}(2 \lambda+1) !} \quad \text { where } \lambda=\left[\frac{1+\sqrt{16 l+17}}{4}\right] .
$$

S. Chaładus and Yu. Teterin prove in the forthcoming paper [2] that the exponent $(k-l) /(k-m)$ in the definition of $c_{0}(k, l, m)$ is the correct one, that is, for any smaller exponent the corresponding supremum is infinite. Moreover they give an estimate for $c_{0}(k, l, m)$ that depends on $k$ and is better than (1) for $k=o\left(l^{2}\right)$. 
Let us note that for large $l$ the minimum on the right-hand side of $(1)$ is equal to the first term for $m<c_{1} l / \log l$, to the last term for $m>c_{2} l$, where $c_{1}, c_{2}$ are suitable constants, $c_{1}>0, c_{2}<1$, provided in the latter case that $\gamma_{l}, \log \left(g_{0}(l) \kappa_{l} / 2^{l}\right)$ are regularly growing functions and

$$
\liminf _{l \rightarrow \infty} \frac{\log g_{0}(l)-\frac{l}{2} \log \gamma_{l}}{l}>\frac{1}{2}
$$

For $m=1,(1)$ constitutes an improvement over [8, Theorem 1] already for $l>50$. The problem of existence of a bound for $c_{0}(k, l, m)$ depending only on $m$ remains open also for $m=1$.

LEMMA 1. If $\mathbf{A}$ is a parallelohedron given by the inequalities

$$
\left|\mathbf{a}_{i} \mathbf{x}\right| \leq 1, \quad \mathbf{a}_{i} \in \mathbb{R}^{l} \quad(1 \leq i \leq k)
$$

then for every parallelopiped $\mathbb{P}$ containing $\mathbf{A}$, symmetric with respect to $\mathbf{0}$ and for a suitable subset $S$ of $\{1,2, \ldots, k\}$ of cardinality $l$ we have

$$
\operatorname{vol} \mathbb{P} \geq \operatorname{vol} \mathbb{P}_{0}(S)
$$

where $\mathbb{P}_{0}(S)$ is the parallelopiped

$$
\left|\mathbf{a}_{i} \mathbf{x}\right| \leq 1 \quad(i \in S) .
$$

Proof. We shall proceed by induction on the number $n$ of pairs of parallel $(l-1)$ dimensional faces of $\mathbb{P}$ that do not contain $(l-1)$ dimensional faces of $\mathbf{A}$ (in the sequel, briefly, faces). If $n=0$ the assertion is true. Suppose it is true for the case of $n-1$ pairs of parallel faces and consider a parallelopiped $\mathbb{P}$ symmetric with respect to 0 with exactly $n$ pairs of parallel faces not containing faces of $\mathbf{A}$. Let $\mathbb{P}$ be given by the inequalities

$$
\left|\mathbf{b}_{i} \mathbf{x}\right| \leq 1, \quad \mathbf{b}_{i} \in \mathbb{R}^{l} \quad(1 \leq i \leq l)
$$

and let $\mathbf{b}_{i} \mathbf{x}= \pm 1$ be the pair of hyperplanes corresponding to one of the $n$ pairs in question. Replacing $\mathbb{P}$ if necessary by a smaller parallelopiped we may assume that there is $x_{0} \in A$ such that

$$
\mathbf{b}_{1} \mathbf{x}_{0}=1 \text {. }
$$

Let $I=\left\{i \leq k:\left|\mathbf{a}_{i} \mathbf{x}_{0}\right|=1\right\}$ and let

$$
\mathbf{a}_{i} \mathbf{x}_{0}=\varepsilon_{i} \quad(i \in I) .
$$

From the fact that the hyperplane $b_{l} x=1$ is supporting $A$ at $x_{0}$ it follows that

$$
\varepsilon_{i} \mathbf{a}_{i} \mathbf{t} \leq 0 \quad(i \in I) \text { implies } \mathbf{b}_{i} \mathbf{t} \leq 0 \text { for } \mathbf{t} \in \mathbb{R}^{l}
$$


Indeed, suppose for some $\mathbf{t}_{0} \in \mathbb{R}^{l}$ that $\varepsilon_{i} \mathbf{a}_{i} \mathbf{t}_{0} \leq 0$ and $\mathbf{b}_{1} \mathbf{t}_{0}>0$. Then for

$$
\mathbf{t}_{1}=\frac{\mathbf{t}_{0}}{\ln \left(\mathbf{t}_{0}\right)} \min \left\{\min _{i \notin I} \frac{1-\left|\mathbf{a}_{i} \mathbf{x}_{0}\right|}{h\left(\mathbf{a}_{i}\right)}, \min _{i \in I} \frac{2}{h\left(\mathbf{a}_{i}\right)}\right\}
$$

we have $\pm\left(\mathbf{x}_{0}+\mathbf{t}_{1}\right) \in A, \mathbf{b}_{1}\left(\mathbf{x}_{0}+\mathbf{t}_{1}\right)>1, \mathbf{b}_{1}\left(-\mathbf{x}-\mathbf{t}_{1}\right)<-1<1$, and thus the hyperplane $b_{1} x=1$ divides $A$. This contradiction proves (4). Hence by a theorem of Farkas [5, page 5] (I owe this reference to Professor S. Rolewicz. There is a related earlier statement in [8, page 45]) we have

$$
\mathbf{b}_{1}=\sum_{i \in I} \varepsilon_{i} \mathbf{a}_{i} \lambda_{i},
$$

where

$$
\lambda_{i} \geq 0 \quad(i \in I)
$$

and by (2) and (3)

$$
\sum_{i \in I} \lambda_{i}=1
$$

Therefore,

$$
\begin{aligned}
(\operatorname{vol} \mathbb{P})^{-1} & =2^{-l}\left|\operatorname{det}\left(\sum_{i \in I} \varepsilon_{i} \mathbf{a}_{i} \lambda_{i}, \mathbf{b}_{2}, \ldots, \mathbf{b}_{l}\right)\right| \\
& =2^{-l}\left|\sum_{i \in I} \lambda_{i} \operatorname{det}\left(\varepsilon_{i} \mathbf{a}_{i}, \mathbf{b}_{2}, \ldots, \mathbf{b}_{l}\right)\right| .
\end{aligned}
$$

Regarding $\lambda_{i}$ as variables restricted by the conditions (5) and (6), we easily see that the right-hand side of (7) takes the maximum for $\lambda_{i}=1$ if $i=i_{0}$, $\lambda_{i}=0$ otherwise. Hence

$$
\operatorname{vol} \mathbb{P} \geq \operatorname{vol} \mathbb{P}_{1},
$$

where $\mathbb{P}_{1}$ is the parallelopiped

$$
\left|\mathbf{a}_{i_{0}} \mathbf{x}\right| \leq 1, \quad\left|\mathbf{b}_{i} \mathbf{x}\right| \leq 1 \quad(2 \leq i \leq l) .
$$

However $\mathbb{P}_{1}$ contains $\mathbf{A}$ and it has only $n-1$ pairs of parallel faces that do not contain faces of $\mathbf{A}$. Thus by the inductive assumption there exists a set $S \subset\{1,2, \ldots, k\}$ of cardinality $l$ and with the property

$$
\operatorname{vol} \mathbb{P}_{1} \geq \operatorname{vol} \mathbb{P}_{0}(S) \text {. }
$$

In view of (8) this gives

$$
\operatorname{vol} \mathbb{P} \geq \operatorname{vol} \mathbb{P}_{0}(S)
$$

and concludes the inductive argument. 
Lemma 2. For all linearly independent vectors $\mathbf{c}_{1}, \ldots, \mathbf{c}_{l} \in \mathbb{R}^{k}$ the domain

$$
\mathbb{C}: h\left(\mathbf{c}_{1} x_{1}+\cdots+\mathbf{c}_{l} x_{l}\right) \leq 1
$$

satisfies

$$
\operatorname{vol} \mathbb{C} \geq \frac{2^{l}}{g_{0}(l) H\left(\mathbf{c}_{1}, \ldots, \mathbf{c}_{l}\right)}, \quad \operatorname{vol} \mathscr{E}(\mathbb{C}) \geq \frac{\kappa_{l}}{g_{1}(l) H\left(\mathbf{c}_{1}, \ldots, \mathbf{c}_{l}\right)} .
$$

Proof. Put

$$
\mathbf{a}_{i}=\left[c_{1 i}, c_{2 i}, \ldots, c_{l i}\right] \quad(1 \leq i \leq k)
$$

Then

$$
\mathbb{C}=\left\{x \in \mathbb{R}^{l}:\left|\mathbf{a}_{i} \mathbf{x}\right| \leq 1 \text { for all } i \leq k\right\}
$$

and clearly $\mathbb{C}$ is a convex body symmetric with respect to $\mathbf{0}$. By Definition 2

$$
\operatorname{vol} \mathbb{C} \geq g_{0}(l)^{-1} \text { inf vol } \mathbb{P}, \quad \operatorname{vol} \mathscr{E}(\mathbb{C}) \geq g_{1}(l)^{-1} 2^{-l} \kappa_{l} \text { inf vol } \mathbb{P},
$$

where the infimum is taken over all parallelopipeds $\mathbb{P}$ symmetric with respect to $\mathbf{0}$ and containing $\mathbb{C}$. However by Lemma 1 the infimum can be replaced by the minimum taken over the finite set of all parallelopipeds

$$
\mathbb{P}_{0}(S), \quad\left|\mathbf{a}_{i} \mathbf{x}\right| \leq 1 \quad(i \in S),
$$

where $S$ runs through all subsets of $\{1, \ldots, k\}$ of cardinality $l$. Since

$$
\operatorname{vol} \mathbb{P}_{0}(S)=2^{l}\left|\operatorname{det}\left\{\mathbf{a}_{i}: i \in S\right\}\right|^{-1}
$$

we have by (9) that

$$
\min \operatorname{vol} \mathbb{P}_{0}(S)=2^{l} H\left(\mathbf{c}_{1}, \ldots, \mathbf{c}_{l}\right)^{-1}
$$

and the lemma follows.

LEMMA 3. If for all linearly independent vectors $\mathbf{n}_{1}, \ldots, \mathbf{n}_{m} \in \mathbb{Z}^{k}$ such that $D\left(\mathbf{n}_{1}, \ldots, \mathbf{n}_{m}\right)=1$ there exist linearly independent vectors $\mathbf{p}_{1}, \ldots, \mathbf{p}_{l} \in \mathbb{Z}^{k}$ such that

$$
\mathbf{n}_{i}=\sum_{j=1}^{l} u_{i j} \mathbf{p}_{j}, \quad u_{i j} \in \mathbb{Q}
$$

and

$$
\prod_{j=1}^{l} h\left(\mathbf{p}_{j}\right) \leq c H\left(\mathbf{n}_{1}, \ldots, \mathbf{n}_{m}\right)^{(k-l) /(k-m)}
$$

then $c_{0}(k, l, m) \leq c$.

Proof. Consider $m$ linearly independent vectors $\mathbf{n}_{1}, \ldots, \mathbf{n}_{m} \in \mathbb{Z}^{k}$ and let $\mathscr{N}$ be the linear space spanned by them over $\mathbb{R}$. Further, let $\mathbf{b}_{1}, \ldots, \mathbf{b}_{m}$ 
be a basis of the lattice $\mathscr{N} \cap \mathbb{Z}^{k}$ and $\mathbf{c}_{1}, \ldots, \mathbf{c}_{k-m} \in \mathbb{Z}^{k}$ linearly independent vectors perpendicular to $\mathscr{N}$. Since $\mathscr{N} \cap \mathbb{Z}^{k}$ is the lattice of all solutions $\mathbf{x} \in \mathbb{Z}^{k}$ of the system $\mathbf{c}_{i} \mathbf{x}=0 \quad(1 \leq i \leq k-m)$, we have by the known theorem [3, page 53] that

$$
D\left(\mathbf{b}_{1}, \ldots, \mathbf{b}_{m}\right)=1 .
$$

On the other hand clearly

$$
\left(\begin{array}{c}
\mathbf{n}_{1} \\
\vdots \\
\mathbf{n}_{m}
\end{array}\right)=\mathbb{A}\left(\begin{array}{c}
\mathbf{b}_{1} \\
\vdots \\
\mathbf{b}_{m}
\end{array}\right),
$$

where $A$ is an integral square matrix of order $m$. It follows from (11) that

$$
\begin{gathered}
D\left(\mathbf{n}_{1}, \ldots, \mathbf{n}_{m}\right)=|\operatorname{det} \mathbf{A}| D\left(\mathbf{b}_{1}, \ldots, \mathbf{b}_{m}\right), \\
H\left(\mathbf{n}_{1}, \ldots, \mathbf{n}_{m}\right)=|\operatorname{det} \mathbf{A}| H\left(\mathbf{b}_{1}, \ldots, \mathbf{b}_{m}\right)
\end{gathered}
$$

and by (10)

$$
H\left(\mathbf{b}_{1}, \ldots, \mathbf{b}_{m}\right)=\frac{H\left(\mathbf{n}_{1}, \ldots, \mathbf{n}_{m}\right)}{D\left(\mathbf{n}_{1}, \ldots, \mathbf{n}_{m}\right)} .
$$

By the assumption of the lemma there exist linearly independent vectors $\mathbf{p}_{1}, \ldots, \mathbf{p}_{l} \in \mathbb{Z}^{k}$ and a matrix $\mathbb{U} \in \mathscr{M}_{m, l}(\mathbb{Q})$ such that

$$
\left(\begin{array}{c}
\mathbf{b}_{1} \\
\vdots \\
\mathbf{b}_{m}
\end{array}\right)=\mathbb{U}\left(\begin{array}{c}
\mathbf{p}_{1} \\
\vdots \\
\mathbf{p}_{l}
\end{array}\right)
$$

and

$$
\prod_{j=1}^{l} h\left(\mathbf{p}_{j}\right) \leq c H\left(\mathbf{b}_{1}, \ldots, \mathbf{b}_{m}\right)^{(k-l) /(k-m)} .
$$

It follows from (11) and (13) that

$$
\left(\begin{array}{c}
\mathbf{n}_{1} \\
\vdots \\
\mathbf{n}_{m}
\end{array}\right)=\mathbb{A} \mathbb{U}\left(\begin{array}{c}
\mathbf{p}_{1} \\
\vdots \\
\mathbf{p}_{l}
\end{array}\right)
$$

while from (12) and (14) that

$$
\prod_{j=1}^{l} h\left(\mathbf{p}_{j}\right) \leq\left(\frac{H\left(\mathbf{n}_{1}, \ldots, \mathbf{n}_{m}\right)}{D\left(\mathbf{n}_{1}, \ldots, \mathbf{n}_{m}\right)}\right)^{(k-l) /(k-m)} .
$$

Thus by Definition $1, c_{0}(k, l, m) \leq c$. 
LEMMA 4. Let $\mathbb{K}$ be a convex domain symmetric with respect to 0 in the linear subspace $\mathscr{L}: x_{1}=\cdots=x_{m}=0$ of $\mathbb{R}^{k}$, not containing in its interior any point of the lattice $\mathscr{L} \cap \mathbb{Z}^{k}$ except $\mathbf{0}$ and let \|\|$_{\mathrm{K}}$ be the corresponding distance function. Let $\mathbf{n}_{1}, \ldots, \mathbf{n}_{m} \in \mathbb{Z}^{k}$ and $\mathscr{N}$ be the linear space spanned by $\mathbf{n}_{1}, \ldots, \mathbf{n}_{m}$ over $\mathbb{R}$. If $\Delta=\operatorname{det}\left(n_{i j}\right)_{i, j \leq m} \neq 0$ and $D\left(\mathbf{n}_{1}, \ldots, \mathbf{n}_{m}\right)=$ 1 there exist vectors $\mathbf{n}_{m+1}, \ldots, \mathbf{n}_{k} \in \mathbb{Z}^{k}$ such that $\mathbf{n}_{1}, \ldots, \mathbf{n}_{k}$ are linearly independent and

$$
\prod_{i=m+1}^{k}\left\|\left(\mathbf{n}_{i}+\mathscr{N}\right) \cap \mathscr{L}\right\|_{\mathbf{K}} \leq 2^{k-m}(\operatorname{vol} \mathbb{K})^{-1}|\Delta|^{-1}
$$

ReMARK. Since $\Delta \neq 0$ we have $\mathscr{N} \cap \mathscr{L}=\{0\}$, and hence $\left(\mathbf{n}_{i}+\mathscr{N}\right) \cap \mathscr{L}$ consists of one point and $\left\|\left(\mathbf{n}_{i}+\mathscr{N}\right) \cap \mathscr{L}\right\|_{\mathbf{K}}$ means the distance from this point to 0 measured through $\mathbb{K}$.

Proof. If $|\Delta|=1$ the desired conclusion follows directly from Minkowski's second theorem. Indeed by that theorem applied to the domain $\mathbb{K}$ there exist linearly independent vectors $\mathbf{n}_{m+1}, \ldots, \mathbf{n}_{k} \in \mathscr{L} \cap \mathbb{K}$ such that

$$
\prod_{i=m+1}^{k}\left\|\mathrm{n}_{i}\right\|_{\mathrm{K}} \leq 2^{k-m}(\operatorname{vol} \mathbb{K})^{-1}
$$

Since $\mathscr{N} \cap \mathscr{L}=\{\boldsymbol{0}\}$ we have $\left(\mathbf{n}_{i}+\mathscr{N}\right) \cap \mathscr{L}=\left\{\mathbf{n}_{i}\right\} \quad(m<i \leq k)$ and $\mathbf{n}_{1}, \ldots, \mathbf{n}_{k}$ are linearly independent. Therefore assume that $|\Delta|>1$. Let $\Delta_{i}(\mathbf{x})$ be the determinant of the matrix obtained from $\left(n_{i j}\right)_{i, j \leq m}$ by replacing the $i$ th row by the first $m$ coordinates of the vector $\mathbf{x}$.

Let us take a real number $r>1$ and consider in $\mathbb{R}^{k}$ the domain

$$
\mathbb{D}_{r}(\mathbb{K}): \max _{1 \leq \mu \leq m}\left|\Delta_{\mu}(\mathbf{x})\right|+|\Delta|^{r}\left\|\mathbf{x} \Delta-\sum_{\mu=1}^{m} \mathbf{n}_{\mu} \Delta_{\mu}(\mathbf{x})\right\|_{\mathbf{K}}^{(k-m) r} \leq|\Delta|^{(k-m) r} .
$$

Then $\mathbb{D}_{r}(\mathbb{K})$ is convex and symmetric with respect to 0 . In order to compute its volume we make the affine transformation

$$
\frac{\Delta_{\mu}(\mathbf{x})}{\Delta^{(k-m) r}}=y_{\mu} \quad(\mu=1, \ldots, m), \quad x_{\mu}=y_{\mu} \quad(\mu=m+1, \ldots, k) .
$$

This transformation has Jacobian equal to $\Delta^{(k-m) r m-m+1}$ and it transforms $\mathbb{D}_{r}(\mathbf{K})$ into

$$
\mathbb{D}_{r}^{\prime}(\mathbb{K}): \max _{1 \leq \mu \leq m}\left|y_{\mu}\right|+|\Delta|^{r}\left\|\left[0, y_{m+1}, \ldots, y_{k}\right]-\sum_{\mu=1}^{m} \mathbf{n}_{\mu}^{\prime} y_{\mu} \Delta^{(k-m) r-1}\right\|_{\mathbf{K}}^{(k-m) r} \leq 1,
$$


where $\mathbf{n}_{\mu}^{\prime}$ is the projection of $\mathbf{n}_{\mu}$ on $\mathscr{L}$. Clearly

$$
\begin{aligned}
\operatorname{vol} \mathbb{D}_{r} & (\mathbb{K})=|\Delta|^{(k-m) r m-m+1} \operatorname{vol} \mathbb{D}_{r}^{\prime}(\mathbb{K}) \\
= & |\Delta|^{(k-m) r m-m+1} \operatorname{vol} \mathbb{K} \int_{\max _{1 \leq \mu \leq m}\left|y_{\mu}\right| \leq 1} d y_{1} d y_{2} \cdots d y_{m}\left(\frac{1-\max \left|y_{\mu}\right|}{|\Delta|^{r}}\right)^{1 / r} \\
& =2^{m}|\Delta|^{((k-m) r-1) m} \operatorname{vol} \mathbb{K} \int_{0}^{1} m t^{m-1}(1-t)^{1 / r} d t .
\end{aligned}
$$

Put $\int_{0}^{1} m t^{m-1}(1-t)^{1 / r} d t=I_{r, m}$.

Let $\lambda_{i}=\inf \left\{\lambda: \operatorname{dim} \lambda \mathbb{D}_{r}(\mathbb{K}) \cap \mathbb{Z}^{k} \geq i\right\} \quad(1 \leq i \leq k)$. By Minkowski's second theorem there exist linearly independent points $\mathbf{m}_{1}, \ldots, \mathbf{m}_{k}$ such that

$$
\mathbf{m}_{i} \in \lambda_{i} \mathbb{D}_{r}(\mathbb{K}) \cap \mathbb{Z}^{k}
$$

and

$$
\prod_{i=1}^{k} \lambda_{i} \leq 2^{k} \operatorname{vol} \mathbb{D}_{r}(\mathbb{K})^{-1}=2^{k-m} I_{r, m}^{-1}(\operatorname{vol} \mathbb{K})^{-1}|\Delta|^{(1-(k-m) r) m} .
$$

We shall show that

$$
\lambda_{i}=|\Delta|^{1-(k-m) r} \quad(1 \leq i \leq m)
$$

and

$$
\mathbf{m}_{i} \in \mathscr{N} \quad(1 \leq i \leq m) .
$$

Indeed, for $i \leq m, \mu \leq m$ we have

$$
\begin{gathered}
\Delta_{\mu}\left(\mathbf{n}_{i}\right)=\Delta \quad \text { if } \mu=i, \quad 0 \text { otherwise; } \\
\Delta \mathbf{n}_{i}=\sum_{\mu=1}^{m} \mathbf{n}_{\mu} \Delta_{\mu}\left(\mathbf{n}_{i}\right),
\end{gathered}
$$

and hence

$$
\mathbf{n}_{i} \in|\Delta|^{1-(k-m) r} \mathbb{D}_{r}(\mathbb{K}) \quad(1 \leq i \leq m) .
$$

On the other hand, if $\mathbf{x} \in \lambda \mathbb{D}_{r}(\mathbb{K}) \cap \mathbb{Z}^{k}$ and $\mathbf{x} \notin \mathscr{N}$ we have $\Delta \mathbf{x} \neq$ $\sum_{\mu=1}^{m} \mathbf{n}_{\mu} \Delta_{\mu}(\mathbf{x})$, and thus by the assumption about $\mathbb{K},\left\|\Delta \mathbf{x}-\sum_{\mu=1}^{n_{\mu}} \mathbf{n}_{\mu} \Delta_{\mu}(\mathbf{x})\right\|_{\mathbf{K}}$ $\geq 1$ and by the definition of $\mathbb{D}_{r}(\mathbb{K})$,

$$
\lambda^{(k-m) r}|\Delta|^{(k-m) r} \geq|\Delta|^{r} ; \quad \lambda \geq|\Delta|^{-1+\frac{1}{k-m}}>|\Delta|^{1-(k-m) r} .
$$

If $\mathbf{x} \in \lambda \mathbb{D}_{r}(\mathbb{K}) \cap \mathbb{Z}^{k}$ and $\mathbf{x} \in \mathscr{N}$ we have $\Delta \mathbf{x}=\sum_{\mu=1}^{m} \mathbf{n}_{\mu} \Delta_{\mu}(\mathbf{x})$ and thus by the assumption that $D\left(\mathbf{n}_{1}, \ldots, \mathbf{n}_{m}\right)=1$ we have $\Delta_{\mu}(\mathbf{x}) \equiv 0(\bmod \Delta)$, and 
hence either $\mathbf{x}=\mathbf{0}$ or $\max _{1 \leq \mu \leq m}\left|\Delta_{\mu}(\mathbf{x})\right| \geq|\Delta|$, which by the definition of $\mathbb{D}_{r}(\mathbb{K})$ implies

$$
\lambda \geq|\Delta|^{1-(k-m) r} .
$$

The claims (17) and (18) follow from (19), (20) and (21).

From (16) and (17) we infer that

$$
\prod_{i=m+1}^{k} \lambda_{i} \leq 2^{k-m}(\operatorname{vol} \mathbb{K})^{-1} I_{r, m}^{-1}
$$

and since by (15)

$$
|\Delta|^{r}\left\|\Delta \mathbf{m}_{i}-\sum_{\mu=1}^{x} \mathbf{n}_{\mu} \Delta_{\mu}\left(\mathbf{m}_{i}\right)\right\|_{\mathbf{K}}^{(k-m) r} \leq|\Delta|^{(k-m) r} \lambda_{i}^{(k-m) r}
$$

we obtain

$$
\prod_{i=m+1}^{k}\left\|\mathbf{m}_{i}-\Delta^{-1} \sum_{\mu=1}^{m} \mathbf{n}_{\mu} \Delta_{\mu}\left(\mathbf{m}_{i}\right)\right\|_{\mathbf{K}} \leq 2^{k-m}(\operatorname{vol} \mathbb{K})^{-1}|\Delta|^{-1} I_{r, m}^{-1} .
$$

Moreover, by (18), $\mathbf{n}_{1}, \ldots, \mathbf{n}_{m}, \mathbf{m}_{m+1}, \ldots, \mathbf{m}_{k}$ are linearly independent. For every $r>1$ there corresponds a certain choice of vectors $\mathbf{m}_{i} \in \mathbb{Z}^{k}$, however the set of values which we can obtain on the left-hand side of (22) is discrete. Therefore there exist vectors $\mathbf{n}_{i}(m<i \leq k)$ such that $\mathbf{n}_{i}$ $(1 \leq i \leq k)$ are linearly independent and

$$
\prod_{i=m+1}^{k}\left\|\mathbf{n}_{i}-\Delta^{-1} \sum_{\mu=1}^{m} \mathbf{n}_{\mu} \Delta_{\mu}\left(\mathbf{n}_{i}\right)\right\|_{\mathbf{K}} \leq 2^{k-m}(\operatorname{vol} \mathbb{K})^{-1}|\Delta|^{-1} \lim _{r \rightarrow \infty} I_{r, m}^{-1} .
$$

However

$$
\left\{\mathbf{n}_{i}-\Delta^{-1} \sum_{\mu=1}^{m} \mathbf{n}_{\mu} \Delta_{\mu}\left(\mathbf{n}_{i}\right)\right\}=\left(\mathbf{n}_{i}+\mathscr{N}\right) \cap \mathscr{L}
$$

and

$$
\lim _{r \rightarrow \infty} I_{r, m}=\int_{0}^{1} m t^{m-1} d t=1,
$$

which proves the lemma.

Lemma 5. If $m<k, \mathbf{n}_{1}, \ldots, \mathbf{n}_{m} \in \mathbb{Z}^{k}, D\left(\mathbf{n}_{1}, \mathbf{n}_{2}, \ldots, \mathbf{n}_{m}\right)=1$ there exist vectors $\mathbf{n}_{m+1}, \ldots, \mathbf{n}_{k} \in \mathbb{Z}^{k}$ such that $\mathbf{n}_{1}, \ldots, \mathbf{n}_{k}$ are linearly independent and for each $l \in[m, k]$ the domain $\mathbb{D} \subset \mathbb{R}^{l}: h\left(\sum_{i=1}^{l} x_{i} \mathbf{n}_{i}\right) \leq 1$ satisfies

$$
\operatorname{vol} \mathbb{D} \geq \frac{2^{l} m !}{g_{0}(m) l !} H\left(\mathbf{n}_{1}, \ldots, \mathbf{n}_{m}\right)^{-(k-l) /(k-m)}
$$


and

$$
\begin{aligned}
\operatorname{vol} \mathscr{E}(\mathbb{D}) \geq \max \left\{\frac{\kappa_{l}}{g_{1}(m)(l-m+1)^{l / 2}},\right. & \left.\frac{\kappa_{l}}{g_{1}(l)}\left(\begin{array}{c}
l \\
m
\end{array}\right)^{-1 / 2} l^{(m-l) / 2}\right\} \\
& \times H\left(\mathbf{n}_{1}, \ldots, \mathbf{n}_{m}\right)^{(k-l) /(k-m)}
\end{aligned}
$$

Proof. Without loss of generality we may assume that $H\left(\mathbf{n}_{1}, \mathbf{n}_{2}, \ldots, \mathbf{n}_{m}\right)$ $=|\Delta|$, where $\Delta=\operatorname{det}\left(n_{i j}\right)_{i, j \leq m}$. By Lemma 4 applied with $\mathbb{K}=\left\{\mathbf{x} \in \mathscr{L}^{\prime}\right.$ : $h(\mathbf{x}) \leq 1\}$ there exists vectors $\mathbf{n}_{m+1}, \ldots, \mathbf{n}_{k} \in \mathbb{Z}^{k}$ such that $\mathbf{n}_{1}, \ldots, \mathbf{n}_{l}$ are linearly independent and

$$
\prod_{i=m+1}^{k} h\left(\mathbf{n}_{i}^{\prime}\right) \leq|\Delta|^{-1}, \quad \text { where }\left\{\mathbf{n}_{i}^{\prime}\right\}=\left(\mathbf{n}_{i}+\mathscr{N}\right) \cap \mathscr{L} \quad(m<i \leq k) .
$$

Permuting the vectors $\mathbf{n}_{i}$ if necessary we may assume that the sequence $h\left(\mathbf{n}_{i}^{\prime}\right)$ is nondecreasing. Then (25) implies

$$
\prod_{i=m+1}^{l} h\left(\mathbf{n}_{i}^{\prime}\right) \leq H\left(\mathbf{n}_{1}, \ldots, \mathbf{n}_{m}\right)^{-(l-m) /(k-m)} .
$$

In order to prove (23) let us write explicitly

$$
\mathbf{n}_{i}^{\prime}=\mathbf{n}_{i}-\sum_{\mu=1}^{m} a_{i \mu} \mathbf{n}_{\mu} \quad(m<i \leq l) .
$$

Then

$$
\sum_{i=1}^{l} x_{i} \mathbf{n}_{i}=\sum_{\mu=1}^{m} \mathbf{n}_{\mu}\left(x_{\mu}+\sum_{i=m+1}^{l} a_{i \mu} x_{i}\right)+\sum_{i=m+1}^{l} x_{i} \mathbf{n}_{i}^{\prime}
$$

and

$$
h\left(\sum_{i=1}^{l} x_{i} \mathbf{n}_{i}\right) \leq h\left(\sum_{\mu=1}^{m} \mathbf{n}_{\mu}\left(x_{\mu}+\sum_{i=m+1}^{l} a_{i \mu} x_{i}\right)\right)+\sum_{i=m+1}^{l}\left|x_{i}\right| h\left(\mathbf{n}_{i}^{\prime}\right) .
$$

It follows by a change of variables that

$$
\begin{array}{r}
\operatorname{vol} \mathbb{D} \geq \int_{\mathbf{D}_{0}} d x_{m+1} \cdots d x_{l} \text { vol }\left\{\mathbf{x} \in \mathbb{R}^{m}: h\left(\sum_{\mu=1}^{m} x_{\mu} \mathbf{n}_{\mu}\right)\right. \\
\left.\leq 1-\sum_{i=m+1}^{l}\left|x_{i}\right| h\left(\mathbf{n}_{i}^{\prime}\right)\right\},
\end{array}
$$

where $\mathbb{D}_{0}$ is the domain $\sum_{i=m+1}^{l}\left|x_{i}\right| h\left(\mathbf{n}_{i}^{\prime}\right) \leq 1$. However by Lemma 2,

$$
\operatorname{vol}\left\{\mathbf{x} \in \mathbb{R}^{m}: h\left(\sum_{\mu=1}^{m} x_{\mu} \mathbf{n}_{\mu}\right) \leq c\right\} \geq \frac{2^{m} c^{m}}{g_{0}(m) H\left(\mathbf{n}_{1}, \ldots, \mathbf{n}_{m}\right)},
$$


and hence

$$
\begin{aligned}
\operatorname{vol} \mathbb{D} & =\frac{2^{m}}{g_{0}(m) H\left(\mathbf{n}_{1}, \ldots, \mathbf{n}_{m}\right)} \int_{\mathbf{D}_{0}}\left(1-\sum_{i=m+1}^{l}\left|x_{i}\right| h\left(\mathbf{n}_{i}^{\prime}\right)\right)^{m} d x_{m+1} \cdots d x_{l} \\
& =\frac{2^{l} m !}{g_{0}(m) l ! H\left(\mathbf{n}_{1}, \ldots, \mathbf{n}_{m}\right)} \prod_{i=m+1}^{l} h\left(\mathbf{n}_{i}^{\prime}\right)^{-1}
\end{aligned}
$$

and (23) follows from (26).

In order to prove the part of (24) corresponding to the first term of the maximum on the right-hand side, let $\mathbb{D}_{1}$ be the domain $h\left(\sum_{i=1}^{m} x_{i} \mathbf{n}_{i}\right) \leq 1$. The ellipsoid $\mathscr{E}\left(\mathbb{D}_{1}\right)$ is given by the inequality $F_{1}\left(x_{1}, \ldots, x_{m}\right) \leq 1$, where $F_{1}$ is a positive definite quadratic form.

Since $\mathscr{E}\left(\mathbb{D}_{1}\right) \subset \mathbb{D}_{1}$ we have for all $\mathbf{x} \in \mathbb{R}^{m}$,

$$
\sqrt{F_{1}\left(x_{1}, \ldots, x_{m}\right)}=\|\mathbf{x}\|_{\mathscr{E}\left(\mathbf{D}_{1}\right)} \geq\|\mathbf{x}\|_{\mathbf{D}_{1}}=h\left(\sum_{i=1}^{m} x_{i} \mathbf{n}_{i}\right) .
$$

By virtue of Lemma 2, we have

$$
\operatorname{vol} \mathscr{E}\left(\mathbb{D}_{1}\right) \geq \kappa_{m} g_{1}(m)^{-1} H\left(\mathbf{n}_{1}, \ldots, \mathbf{n}_{m}\right)^{-1} .
$$

However

and thus

$$
\operatorname{vol} \mathscr{E}\left(\mathbb{D}_{1}\right)=\frac{\kappa_{m}}{\sqrt{d\left(F_{1}\right)}}
$$

$$
\sqrt{d\left(F_{1}\right)} \leq g_{1}(m) H\left(\mathbf{n}_{1}, \ldots, \mathbf{n}_{m}\right)
$$

Consider now the quadratic form

$$
\begin{aligned}
F\left(x_{1}, \ldots, x_{l}\right)= & (l-m+1) F_{1}\left(\ldots, x_{\mu}+\sum_{i=m+1}^{l} a_{i \mu} x_{i}, \ldots\right) \\
& +(l-m+1) \sum_{i=m+1}^{l} x_{i}^{2} h^{2}\left(\mathbf{n}_{i}^{\prime}\right) .
\end{aligned}
$$

For all $\mathbf{x} \in \mathbb{R}^{l}$ we have by the Cauchy inequality, by (28) and (27), that

$$
\begin{aligned}
\sqrt{F\left(x_{1}, \ldots, x_{l}\right)} \geq & \sqrt{F_{1}\left(\ldots, x_{\mu}+\sum_{i=m+1}^{l} a_{i \mu} x_{i}, \ldots\right)} \\
& +\sum_{i=m+1}^{l}\left|x_{i}\right| h\left(\mathbf{n}_{i}^{\prime}\right) \geq h\left(\sum_{\mu=1}^{m} \mathbf{n}_{\mu}\left(x_{\mu}+\sum_{i=m+1}^{l} a_{i \mu} x_{i}\right)\right) \\
& +\sum_{i=m+1}^{l}\left|x_{i}\right| h\left(\mathbf{n}_{i}^{\prime}\right) \geq h\left(\sum_{i=1}^{l} x_{i} \mathbf{n}_{i}\right)
\end{aligned}
$$


and thus the ellipsoid

$$
E: F\left(x_{1}, \ldots, x_{l}\right) \leq 1
$$

is contained in $\mathbb{D}$ and by the definition of $\mathscr{E}(\mathbb{D})$,

$$
\operatorname{vol} \mathscr{E}(\mathbb{D}) \geq \operatorname{vol} \mathbb{E}=\frac{\kappa_{l}}{\sqrt{d(F)}} .
$$

Since $F$ is obtained from the quadratic form

$$
(l-m+1)\left(F_{1}+\sum_{i=m+1}^{l} x_{i}^{2} h^{2}\left(\mathbf{n}_{i}^{\prime}\right)\right)
$$

by a unimodular substitution, we have

$$
\sqrt{d(F)}=(l-m+1)^{l / 2} \sqrt{d\left(F_{1}\right)} \prod_{i=m+1}^{l} h\left(\mathbf{n}_{i}^{\prime}\right)
$$

and by (26), (29) and (30),

$$
\operatorname{vol} \mathscr{E}(\mathbb{D}) \geq \kappa_{l}(l-m+1)^{-l / 2} H\left(\mathbf{n}_{1}, \ldots, \mathbf{n}_{m}\right)^{-(k-l) /(k-m)} .
$$

In order to prove the remaining part of (24) note that

$$
H\left(\mathbf{n}_{1}, \ldots, \mathbf{n}_{l}\right)=H\left(\mathbf{n}_{1}, \ldots, \mathbf{n}_{m}, \mathbf{n}_{m+1}^{\prime}, \ldots, \mathbf{n}_{l}^{\prime}\right) .
$$

Let $M$ be a minor of order of $l$ of the matrix

$$
\left(\begin{array}{c}
\mathbf{n}_{1} \\
\vdots \\
\mathbf{n}_{m} \\
\mathbf{n}_{m+1}^{\prime} \\
\vdots \\
\mathbf{n}_{l}^{\prime}
\end{array}\right)
$$

and $S$ the set of indices of the columns of $M$. Developing $M$ according to the first $m$ rows we obtain from the Laplace theorem

$$
|M| \leq H\left(\mathbf{n}_{1}, \ldots, \mathbf{n}_{m}\right) \sum\left|M_{j_{1}, \ldots, j_{l-m}}\right|,
$$

where $M_{j_{1}, \ldots, j_{l-m}}$ is the minor of

$$
\left(\begin{array}{c}
\mathbf{n}_{m+1}^{\prime} \\
\vdots \\
\mathbf{n}_{l}^{\prime}
\end{array}\right)
$$

consisting of the columns $j_{1}, \ldots, j_{l-m}$, while $\left\{j_{1}, \ldots, j_{l-m}\right\}$ runs through all subsets of $S$ of cardinality $l-m$. 
By the generalized Hadamard inequality [1, formula (2.6)]

$$
\sum M_{j_{1}, \ldots, j_{l-m}}^{2} \leq \prod_{i=m+1}^{l} \sum_{j \in S} n_{i j}^{\prime 2} \leq l^{l-m} \prod_{i=m+1}^{l} h\left(\mathbf{n}_{i}^{\prime}\right)^{2},
$$

and hence, by the Cauchy inequality,

$$
\sum\left|M_{j_{1}, \ldots, j_{l-m}}\right| \leq\left(\begin{array}{c}
l \\
m
\end{array}\right)^{1 / 2} l^{(l-m) / 2} \prod_{i=m+1}^{l} h\left(\mathbf{n}_{i}^{\prime}\right) .
$$

The inequalities (26), (31) and (32) give

$$
|M| \leq\left(\begin{array}{c}
l \\
m
\end{array}\right)^{1 / 2} l^{(l-m) / 2} H\left(\mathbf{n}_{1}, \ldots, \mathbf{n}_{m}\right)^{(k-l) /(k-m)},
$$

and hence by the arbitrary choice of $M$

$$
H\left(\mathbf{n}_{1}, \ldots, \mathbf{n}_{l}\right) \leq\left(\begin{array}{c}
l \\
m
\end{array}\right)^{1 / 2} l^{(l-m) / 2} H\left(\mathbf{n}_{1}, \ldots, \mathbf{n}_{m}\right)^{(k-l) /(k-m)} .
$$

Now Lemma 2 applied with $\mathbb{C}=\mathbb{D}$ implies

$$
\operatorname{vol} \mathscr{E}(\mathbb{D}) \geq \frac{\kappa_{l}}{g_{1}(l)}\left(\begin{array}{c}
l \\
m
\end{array}\right)^{-1 / 2} l^{(m-l) / 2} H\left(\mathbf{n}_{1}, \ldots, \mathbf{n}_{m}\right)^{-(k-l) /(k-m)} .
$$

Proof of Theorem 1 . Let $\mathbf{n}_{1}, \ldots, \mathbf{n}_{m} \in \mathbb{Z}^{k}$ be linearly independent and $D\left(\mathbf{n}_{1}, \ldots, \mathbf{n}_{m}\right)=1$. Let $\mathbf{n}_{m+1}, \ldots, \mathbf{n}_{l}$ be vectors the existence of which is asserted in Lemma 5 and consider the domain $\mathbb{D}: h\left(\sum_{j=1}^{l} x_{j} \mathbf{n}_{j}\right) \leq 1$. Let

$$
\mu_{i}=\min \left\{\mu: \operatorname{dim} \mu \mathbb{D} \cap \mathbb{Z}^{l} \geq i\right\} \quad(1 \leq i \leq l) .
$$

By Minkowski's second theorem there exist linearly independent vectors $\mathbf{y}_{i}=$ $\left[y_{i 1}, \ldots, y_{i l}\right](1 \leq i \leq l)$ such that

$$
\mathbf{y}_{i} \in \mu_{i} \mathbb{D} \cap \mathbb{Z}^{l}
$$

and

$$
\prod_{i=1}^{l} \mu_{i} \leq 2^{l}(\operatorname{vol} \mathbb{D})^{-1} .
$$

By another theorem of Minkowski (see $[8, \S 51]$ or $[6, \S 18$, Theorem 3$]$ ),

$$
\prod_{i=1}^{l} \mu_{i} \leq \Delta(\mathscr{E}(\mathbb{D}))^{-1}
$$

where $\Delta(\mathscr{E}(\mathbb{D}))$ is the critical determinant of $\mathscr{E}(\mathbb{D})$ and by the definition of the Hermite constant

$$
\Delta(\mathscr{E}(\mathbb{D}))^{-1}=\gamma_{l}^{l / 2} \frac{\kappa_{l}}{\operatorname{vol} \mathscr{E}(\mathbb{D})}
$$


(see [6, formula (37.6)]). Let us put

$$
\mathbf{p}_{i}=\sum_{j=1}^{l} y_{i j} \mathbf{n}_{j} \quad(1 \leq i \leq l) .
$$

It follows from the definition of $\mathbb{D}$ and from (34)-(37) that $h\left(\mathbf{p}_{i}\right)=\mu_{i}$, hence by (34)-(37)

$$
\prod_{i=1}^{l} h\left(\mathbf{p}_{i}\right) \leq \min \left\{2^{l}(\operatorname{vol} \mathbb{D})^{-1}, \gamma_{l}^{l / 2} \kappa_{l}(\operatorname{vol} \mathscr{E}(\mathbb{D}))^{-1}\right\}
$$

and by Lemma 5

$$
\begin{aligned}
\prod_{i=1}^{l} h\left(\mathbf{p}_{i}\right) \leq \min \left\{\frac{l !}{m !} g_{0}(m),(l-m+1)^{l / 2} g_{1}(m) \gamma_{l}^{l / 2},\right. \\
\left.\qquad\left(\begin{array}{c}
l \\
m
\end{array}\right)^{1 / 2} l^{(l-m) / 2} g_{1}(l) \gamma_{l}^{l / 2}\right\} H\left(\mathbf{n}_{1}, \ldots, \mathbf{n}_{m}\right)^{(k-l) /(k-m)} .
\end{aligned}
$$

Moreover since $\mathbf{y}_{1}, \ldots, \mathbf{y}_{l}$ are linearly independent the system (37) can be solved with respect to $n_{1}, \ldots, n_{l}$ and we obtain

$$
\mathbf{n}_{i}=\sum_{j=1}^{l} u_{i j} \mathbf{p}_{j}, \quad u_{i j} \in \mathbb{Q} \quad(1 \leq i \leq l) .
$$

Since $\mathbf{n}_{i}(1 \leq i \leq l)$ are linearly independent so are $\mathbf{p}_{j}(1 \leq j \leq l)$ and we obtain from (37) and Lemma 3 that

$$
\begin{aligned}
& c_{0}(k, l, m) \leq \min \left\{(l-m+1)^{l / 2} g_{1}(m) \gamma_{l}^{l / 2}\right. \\
&\left.\frac{l !}{m !} g_{0}(m),\left(\begin{array}{c}
l \\
m
\end{array}\right)^{1 / 2} l^{\frac{l-m}{2}} g_{1}(l) \gamma_{l}^{l / 2}\right\},
\end{aligned}
$$

which proves the first part of the theorem.

In order to prove the second part let us observe that if $l=m=1$ the righthand side of (38) equals 1 , while it immediately follows from the definition of $c_{0}(k, l, m)$ that $c_{0}(k, 1,1) \geq 1$. If $l=m=2$ the right hand side of (38) equals $\frac{4}{3}$, since

$$
g_{1}(2)=\sqrt{\frac{4}{3}}, \quad \gamma_{2}=\sqrt{\frac{4}{3}}, \quad g_{0}(2) \geq \frac{4}{3} .
$$

On the other hand, consider the following vectors in $\mathbb{Z}^{k} \quad(k \geq 3)$ $\mathbf{n}_{1}=[2 t, 4 t+1,2 t, 0, \ldots, 0], \quad \mathbf{n}_{2}=[4 t-1,2 t,-2 t, 0, \ldots, 0] \quad(t \in \mathbb{N})$. 
We have here

$$
H\left(\mathbf{n}_{1}, \mathbf{n}_{2}\right)=12 t^{2}+2 t, \quad D\left(\mathbf{n}_{1}, \mathbf{n}_{2}\right)=1 .
$$

Hence, if

$$
\mathbf{n}_{i}=\sum_{j=1}^{2} u_{i j} \mathbf{p}_{j}, \quad u_{i j} \in \mathbb{Q}, \mathbf{p}_{j} \in \mathbb{Z}^{k} \quad(1 \leq i, j \leq 2)
$$

we have

$$
\mathbf{p}_{j}=\mathbf{n}_{1} x_{j}+\mathbf{n}_{2} y_{j}, \quad\left[x_{j}, y_{j}\right] \in \mathbb{Z}^{2} \backslash\{\mathbf{0}\} \quad(1 \leq j \leq 2) .
$$

If $x_{j}=y_{j}$ we have $\left|p_{j 2}\right|>6 t$, otherwise $\left|p_{j 3}\right| \geq 2 t$, and thus $h\left(\mathbf{p}_{j}\right) \geq 2 t$ $(1 \leq j \leq 2)$. If for an $\varepsilon>0$ we have

$$
h\left(\mathbf{p}_{1}\right) h\left(\mathbf{p}_{2}\right) \leq\left(\frac{4}{3}-\varepsilon\right) H\left(\mathbf{n}_{1}, \mathbf{n}_{2}\right)=\left(\frac{4}{3}-\varepsilon\right)\left(12 t^{2}+2 t\right)
$$

then for $t>t_{0}(\varepsilon)$

$$
h\left(\mathbf{p}_{1}\right) h\left(\mathbf{p}_{2}\right)<(16-10 \varepsilon) t^{2}
$$

and since $h\left(\mathbf{p}_{j}\right) \geq 2 t$ we obtain $h\left(\mathbf{p}_{j}\right)<(8-5 \varepsilon) t^{2} \quad(1 \leq j \leq 2)$. Hence for $t>t_{1}(\varepsilon)$, by consideration of the first three coordinates of $\mathbf{p}_{j}$

$$
\left|2 x_{j}+4 y_{j}\right| \leq 7, \quad\left|4 x_{j}+2 y_{i}\right| \leq 7, \quad\left|2 x_{j}-2 y_{j}\right| \leq 7 ;
$$

$\left|x_{j}\right| \leq 1,\left|y_{j}\right| \leq 1$ and since $\left[x_{j}, y_{j}\right] \neq[0,0], h\left(\mathbf{p}_{j}\right) \geq 4 t-1 \quad(1 \leq j \leq 2)$. It follows that

$$
h\left(\mathbf{p}_{1}\right) h\left(\mathbf{p}_{2}\right) \geq 16 t^{2}-8 t+1 \text {, }
$$

which for $t>\max \left\{t_{0}(\varepsilon), t_{1}(\varepsilon), \varepsilon^{-1}\right\}$ contradicts (39). This shows that $c_{0}(k, 2,2)=\frac{4}{3}$ and completes the proof of the theorem.

Proof of Theorem 2. The proof does not differ essentially from the proof of [10, Theorem 2]. In formula (14) and in the fourth displayed formula on page 701 there, one has to replace $c_{0}(k, l)$ by $c_{0}(k, l, m)$ and $h(\mathbf{n})^{(k-l) /(k-m)}$ by $\left(\frac{H\left(\mathbf{n}_{1}, \ldots, \mathbf{n}_{m}\right)}{D\left(\mathbf{n}_{1}, \ldots, \mathbf{n}_{m}\right)}\right)^{(k-l) /(k-m)}$.

\section{Note added in proof}

Yu. Teterin has remarked that Lemma 4 holds under a weaker assumption, namely that vol $\mathbb{K}<\infty$. To see this, it suffices to apply the original formulation to the body of $\lambda \mathbb{R}$ for suitable $\lambda$. 


\title{
References
}

[1] E. Bombieri and J. D. Vaaler, 'On Siegel's lemma', Invent Math. 73 (1983), 11-32.

[2] S. Chatadus and Yu. Teterin, 'Note on a decomposition of integer vectors, II', Acta Arith., to appear.

[3] A. Châtelet, Leçons sur la theorie des nombres, Paris, 1913.

[4] A. Dvoretzky and C. A. Rogers, 'Absolute and unconditional convergence in normed linear spaces', Proc. Nat. Acad. Sci. U.S.A. 36 (1950), 192-197.

[5] J. Farkas, 'Über die Theorie der einfachen Ungleichungen', J. Reine Angew. Math. 124 (1902), 1-27.

[6] P. M. Gruber and C. G. Lekkerkerker, Geometry of numbers, Amsterdam, 1987.

[7] F. John, 'Extremum problems with inequalities as subsidiary conditions', Studies and Essays Presented to R. Courant on his 60th Birthday, January 8, 1948, pp. 187-204, New York, 1948.

[8] H. Minkowski, Geometrie der Zahlen, Leipzig 1896, reprint New York, 1953.

[9] A. Pelczyński and S. J. Szarek, 'On parallelopipeds of minimal volume containing a convex symmetric body in $\mathbf{R}^{n}$, Math. Proc. Cambridge Philos. Soc., to appear.

[10] A. Schinzel, 'A decomposition of integer vectors III', Bull. Polish Acad. Sci. Math. 35 (1987), 693-703.

\author{
Instytut Matematyczny Polskiej Akademii Nauk \\ ul. Sniadeckich 8 \\ Skrytka pocztowa $\mathrm{Nr} 137$ \\ 00-950 Warszawa \\ Poland
}

